\title{
Knowledge and Anchoring: Verification of Three Circumstances in Which Knowledge Does Not Interfere with Anchoring
}

\author{
Fu-Yuan Tang, Tom M. Y. Lin \\ Department of Business Administration, National Taiwan University of Science and Technology, Taiwan \\ Email: fuyan.tang@msa.hinet.net
}

How to cite this paper: Tang, F.-Y. and Lin, T.M.Y. (2017) Knowledge and Anchoring: Verification of Three Circumstances in Which Knowledge Does Not Interfere with Anchoring. Open Journal of Social Sciences, 5, 144-164.

https://doi.org/10.4236/jss.2017.57010

Received: May 19, 2017

Accepted: July 10, 2017

Published: July 13, 2017

Copyright (c) 2017 by authors and Scientific Research Publishing Inc. This work is licensed under the Creative Commons Attribution International License (CC BY 4.0).

http://creativecommons.org/licenses/by/4.0/

\begin{abstract}
Many researchers have demonstrated that those people with higher knowledge are less subject to the impact of a given anchor. In real life, however, a situation might be quite complicated and not simply a question of an existing high (or low) anchor. We have designed four tests to demonstrate the relation between knowledge and anchoring. We hold that only in general situations can knowledge interfere with the anchoring effect. Study 1 was used to demonstrate this hypothesis. The research result in turn served as the foundation for our follow-up studies. Based on this foundation, we conducted three experiments to demonstrate that, when faced with a complicated and difficult estimation that must be completed within a short period of time (Study 2), when the source of information possesses a high degree of reliability (Study 3), and when practical experience is lacking (Study 4), the anchoring effect will still exert influences on people with higher knowledge.
\end{abstract}

\section{Keywords}

Anchoring Effect, Selective Accessibility, Knowledge Level, Time Pressure, Complexity

\section{Introduction}

When making a numeric estimate, people may be influenced by a given arbitrary number. Moreover, they may have a close estimate toward the arbitrary value; despite they are fairly inaccurate sometimes. This is the so-called anchoring effect. For decades, researchers have found evidence to demonstrate that the anchoring effect is a robust psychological phenomenon. [1] asked participants to estimate the length of the Mississippi River regardless of the domain. Further, [2] asked participants to estimate the age of something ("Xianglong") regardless of 
whether the subject was real or fictitious. The following examples were considered by previous researchers as demonstrators of the anchoring effect: general knowledge questions [3], estimates of real estate prices [4], estimates of self-efficacy [5], probability assessments [6], decisions about criminal sentences [7], evaluations of lotteries and gambles [8], math problems [9], and negotiation [10]. Moreover, the anchoring effect can occur whether it is an experiment conducted in a lab or an investigation in the field. [11] suggested that participants rely on a biased set of information that they employed when providing their absolute estimate of the target value. Therefore, estimates based on comparison with an anchor tend to assimilate toward the anchor value. [12] argued that as information is employed about a target, people adjust their estimate away from the anchor. These adjustments, however, tend to be insufficient. A third explanation of anchoring has been proposed concerning attitude change. According to this theory, providing an anchor changes someone's attitude toward being more favorable to the particular attributes of that anchor, thereby biasing future answers to have similar characteristics as the anchor. Leading proponents of this theory consider it an alternate explanation in line with prior research on anchoring, adjusting and selective accessibility. [13] asked participants to view arbitrary ID numbers and estimate the number of physicians in the phone book.

Is the anchoring effect as unquestionably impregnable as studies in many areas have been used to demonstrate its actual existence? In fact, the answer to this question seems to be inconclusive. The questions posed are in tandem: What role do knowledge and experience play? Are more knowledgeable people less influenced by anchoring? Do past behavior and experience affect people's judgment? Recently, researchers have posited different arguments. Some researchers have asserted that knowledge does not moderate anchoring, whereas others have different viewpoints. That is, some researchers have suggested that high-knowledge and low-knowledge people are equally biased by irrelevant anchors [14] [15], whereas some are not. For example, [11] demonstrated that knowledge can, and likely often does, moderate anchoring effects, though they do not assume that this will always be the case. Furthermore, [16] revealed that the particular type of knowledge that people possess is an important determinant of their susceptibility to the anchoring effect. Further, [17] concluded that people's judgments of their own recent behaviors are susceptible to numeric anchors and that self-knowledge does not necessarily reduce the influence of anchors.

Since whether knowledge will interfere with anchoring is still inconclusive, we tried to find out the relations between knowledge and anchoring. These study results can help clarify the role that knowledge plays in affecting the anchoring effect under certain circumstances, and provide future researchers with new research directions to study the anchoring effect.

\section{Anchoring Effect}

\subsection{Selective Accessibility}

When making an estimate, people first make a judgment about whether the giv- 
en anchor and the actual value are equal. Because people tend to engage in hypothesis-consistent testing [18], they may presuppose an identity between the given information and the anchor. When arriving at an estimated value, people might base their estimate on biased information; thus, the estimated value that they arrive at tends to assimilate toward the anchor value. [19], in an experiment, first asked participants whether the percentage of African countries in the United Nations was higher or lower than an ostensibly random number (that is, the anchor), which was determined by spinning a wheel of fortune (a number between 0 and 100). The median estimates of the percentage of African countries in the United Nations were 25 and 45 for groups that received 10 and 65 as starting points, respectively. The above studies suggested that anchors cause people to recruit biased pools of information [3] [20]. [20] "selective accessibility" also explained the above phenomenon.

\subsection{Anchoring and Adjustment}

Anchoring and adjustment is a psychological heuristic that influences the manner in which people intuitively assess probabilities. According to this heuristic, people start with an implicitly suggested reference point (the "anchor") and make adjustments to it to reach their estimate. Owing to a large range of plausible estimates, adjustments that start from a low anchor stop at the lower end of this range, whereas adjustments from a high anchor terminate at the upper end of the range [16]. The study by [19] also found that, when participants were unable to fully compute a long sequence of calculations within a short span of time, the median in the estimate they made regarding the ascending sequence $(1 \times 2 \times 3$ $\times 4 \times 5 \times 6 \times 7 \times 8)$ was higher (2250 versus 512$)$ than that regarding the descending sequence $(8 \times 7 \times 6 \times 5 \times 4 \times 3 \times 2 \times 1)$. This indicates that anchoring occurs not only when the starting point is given to the participant but also when the participant bases his/her estimate on the result of some incomplete computation [19].

\subsection{Priming}

Numeric and magnitude priming posits that anchors prime numbers or magnitudes similar to the anchor value [16]. When participants generated their estimates, these primed numbers were more likely to come to mind, thereby influencing their estimates [4] [21], in their experiments on the price of residential real estate, asked participants to estimate the price of a local house based on listing prices the authors gave them that varied from low to high. The listing price acted as an anchor such that the lower it was, the lower the real estate agents' estimates of the appraised value of the house. In this case, the anchor was not completely arbitrary (that is, the listing prices were often based on appraised values, and the owners of the house presumably did not choose the listing price randomly). Thus, it was common for people to use the listing price when forming their judgments [13].

\subsection{Anchoring and Knowledge}

As mentioned above, a determination of whether knowledge or expertise mod- 
erates the anchoring effect is still inconclusive. In a certain arena, if people possess greater knowledge or richer experience, the possibility of the anchoring effect may be lower, which seems to be a reasonable phenomenon. Many studies have found that experts (those with high knowledge, experience, or expertise in some field) are more resistant to the anchoring effect. Knowledge may play a particularly important role in the judgments of one's own recent behaviors. People attend more to self-relevant information and behavior, and self-referential encoding of information, results in better recall [17]. For instance, investors with adequate experience and good records of transactions in the property market would possibly have a better understanding of the housing market in a specific region than an average person. As they engage in their next investment, they may rely on their past transaction records to decide the selling or purchasing price, and the impact on them of any price that deviates from the market price (with high anchoring or low anchoring) would be relatively small. This is because the knowledge and experience they have accumulated from their past transaction records or from the process of buying and selling, have given them a certain understanding of the property price in a specific region. Furthermore, those who have recently purchased a house might also be less influenced by the anchoring effect as they have a better understanding of the property prices of past transactions as a result of their search for related property information in the process of purchasing a house.

In their study that concludes that "knowledge moderates anchoring," [11] investigated anchoring effects in the American football domain, with participants having very low knowledge as compared to those having very high knowledge. First, the participants were told to answer six questions about American football with high or low anchors. Thereafter, they were asked to indicate their self-perceived football knowledge by clicking on a continuous scale with four equally spaced labels: not at all, somewhat, moderately, and extremely. Then, they indicated their level of knowledge about each target question on the same type of scale. The study found that participants with higher knowledge exhibited smaller anchoring effects. This was true regardless of whether knowledge was assessed with self-report or with objective measures of quiz performance.

Further, [22] examined the effects of anchoring on price estimates in Germany during the transition from the deutsche mark to the euro. They found that before the transition, German participants were more susceptible to anchoring when judging prices in euros (with which they were less familiar) than when judging prices in deutsche marks. In contrast, several months after the transition, when Germany had adapted to the new currency, the pattern reversed: participants instead anchored more when using deutsche marks [17].

[23] also found evidence that numeric judgments of past behavior are susceptible to external influence. They provided participants with false feedback about the success on a task that involved determining whether suicide notes were real or fake. After completing the task, participants were first falsely informed that they had either been very successful, somewhat successful, or very 
unsuccessful. The experimenter then informed participants that this feedback was completely false and asked them to estimate how many notes they had actually correctly identified. Despite the experimenter's explanation, participants who were initially told that they had correctly identified more notes subsequently estimated that they had actually successfully identified more notes than had participants who were initially given more negative feedback. Hence, the initial evaluation provided by the experimenter influenced participants' subsequent numeric judgments of their own behaviors.

As the studies mentioned above indicate, it seems that there is adequate evidence to explain that people with high knowledge or experience are not influenced by anchoring. Some researchers, however, have drawn the opposite conclusion. [4] considered real-world setting. After visiting a piece of property currently for sale, real estate agents and undergraduate students were asked to estimate the appraised value of the property, an appropriate advertised selling price, a reasonable price to pay for the house, and the lowest offer they would accept for this house if they were the seller. Both groups of participants were simultaneously exposed to an anchor. Despite the differences in expertise between the two groups, real estate agents and undergraduate students exhibited similar anchoring effects.

[7] asked experienced legal professionals at educational conferences for judges and prosecutors to assess whether the sentence for a defendant would be higher or lower than 1/3 year(s) (low/high anchor). Consequently, participants who were exposed to the high sentencing anchor gave considerably higher sentences ( $M=33.38$ months) than participants who were confronted with a low anchor ( $M=25.43$ months). An analysis of the mean sentencing decisions indicated that judges were clearly influenced by the potential sentence suggested by the journalist. Thus, even the sentencing decisions of experienced legal professionals may indeed be influenced by clearly irrelevant sentencing anchors.

In studies on whether past behaviors will create an anchoring effect on numeric estimate [17], in their study of solver and observer, suggested that no evidence exists that self-knowledge attenuates the effect of numeric anchors: no difference was observed between the anchoring of observers and that of solvers despite the fact that the solvers should have had more knowledge about their own recent behaviors.

Why does knowledge or expertise moderate the anchoring effect? Highknowledge people might be more likely to know the exact answer for a target estimate and thus will ignore the anchor and provide the known estimate [11]. When judging future behavior, people do not have direct self-knowledge to help them avoid anchoring effects. However, when judging past behavior, people may have such self-knowledge and be able to draw on it to counteract anchors [17]. The relation between knowledge and anchoring effects is complex because not all types of knowledge are equally effective at reducing the biasing influences of anchors. Increased knowledge is important, but only the right type of knowledge can reduce bias [16]. 
However, [4] tested in real-world setting and suggested that professional real estate agents, as compared with amateur undergraduate students, were influenced by anchor in estimating residential real estate. In another study, [17] asked participants to complete anagrams and estimate how many they had just been given after exposure to a high anchor, a low anchor, or no anchor. The results suggested that although an anchoring effect emerged, a possible limitation of the study was that participants were not technically tracking their own behaviors. Since they estimated how many anagrams they were given, rather than how many they completed, participants may not have used information about their behaviors when answering the question.

In light of the fact that different studies offer different readings of the relation between knowledge and anchoring, the question to be asked, then, is whether knowledge will interfere with the anchoring effect. As indicated above, those with higher knowledge might have a better grip on the actual answer in their estimate of a target value in comparison to those with lower knowledge. Thus, those with higher knowledge should be less subject to the impact of a given anchor. However, it seems that this will occurs in general situations. In real life, however, the situation might be quite complicated and not simply a question of the existence of a high (or low) anchor. Based on the above reasons, we hold that it is only in general situations that knowledge can interfere with the anchoring effect, and this deduction also becomes our first hypothesis:

$\mathrm{H} 1$ : In general situations, those with higher knowledge will not be subject to the anchoring effect, but people with low level of knowledge will.

Based on the results of Experiment 1, we would like to make a further deduction: does knowledge necessarily interfere with the anchoring effect without exception? In Hypothesis 1, we stipulate that "in general situations," which means in some specific situations, people with higher knowledge, when making their estimation, will also be influenced by a given anchor set and thereby the anchoring effects. Consider this: if one has to resolve a highly complicated and difficult issue in a short amount of time, then could the anchor exert its influence during this time? This is precisely our second hypothesis:

H2: When faced with a complicated and difficult problem that cannot be resolved within a short time frame, there will still be an anchoring effect for those with a high level of knowledge.

Further, as people with higher knowledge have a deeper understanding of the targeted object whose value they are estimating, they may ignore randomly given anchors. They will, rather, take their own knowledge as the basis for the estimation, as they may consider that randomly given anchors possess no value for reference. Thus, in general situations, the anchoring effect will not occur. However, what would happen if the information source of the anchor had a high reference value? Would people with higher knowledge consider the source of this information as more reliable than the knowledge they possess and consequently take it as a point of reference in their estimation? This deduction becomes our third hypothesis: 
H3: In a case where the information source of the anchor possesses a high reference value, then the anchoring effect will take place even among people with higher knowledge.

Finally, it is not uncommon to hear that people are criticized as "armchair strategists" when they are drawing up plans or making decisions. In other words, although some people may have a relatively high level of knowledge, they may also lack much practical experience. Hence, when making a numeric estimate, they will often tend toward the theoretical side and hence overlook the practical situation. This view becomes our fourth hypothesis.

H4: In the case of a lack of sufficient practical experience, the anchoring effect will still occur even among people with higher knowledge.

\section{Present Studies}

We conducted four studies to prove our hypotheses above. In Study 1, we first performed an experiment to confirm the results of past researchers: one's level of knowledge in fact has a moderating effect on the occurrence of anchoring. This research result then served as the foundation for our follow-up studies. On this foundation, we conducted three experiments to demonstrate that, when faced with a complicated and difficult estimation that must be completed within a short period of time (Study 2), when the source of information possesses a high degree of reliability (Study 3), and when practical experience is lacking (Study 4), the anchoring effect will still happen to people with higher knowledge.

\subsection{Study 1}

\subsubsection{Participants}

We successfully interviewed 80 people who had bought property in New Taipei City (Taiwan) during the past year and simultaneously applied for a loan with a bank; we classified them as group A. Simultaneously, this was the first time these participants had bought a house. Those who in group A were randomly selected from twelve branches of four banks located in New Taipei City. Sixteen of total interviewees were not qualified because they had bought one or more houses in their lives. In addition, we interviewed 80 persons who had resided in the same area for over 3 years with no experience in purchasing a house or plans to buy a house within 1 year; we classified them as group B. All participants would receive a cup of black tea. Upon completion, five of the participants would be selected by lot for a Starbucks coffee coupon as a prize.

\subsubsection{Materials}

Our interviews were divided into three parts. The first part invited the participants who experienced in house buying to answer questions on the process of their property search and price negotiations, while those without experience in house buying were invited to share their views on the current real estate market. This part was unrelated to the data we wanted to analyze. Its purposes were mainly to help the participants recall their past house-buying behaviors or focus more on real estate issues as well as guide them toward having greater interest in 
the follow-up questions so that we could obtain answers closer to the reality. The second part was an anchoring test. The third part was for collecting demographic information about the participants.

\subsubsection{Procedure}

In the first part, we first invited the interviewees to share their house-buying experience. If the interviewees of group A (that is, those with higher knowledge) failed to meet the requirements we laid down (to have bought their first home within the last year and done so in New Taipei City), no follow-up actions would be carried out for them and the interview was considered to be invalid. Similarly, if the interviewees of group B (those without house-buying experience within the past year and who had no intention of buying a house in a year) did not meet the requirements we laid down, no follow-up actions were carried out for them.

In the second part, we presented a residential real estate property located in New Taipei City (Taiwan). Information on the house included location, size, type of building, years, and the current condition. Participants were asked to estimate whether the price of the house we presented was higher or lower than New Taiwan dollar 700 (high anchor) versus 400 (low anchor) thousand per unit. Here 1 unit represents 0.325 square meters. Counterbalancing of the anchors did not affect the results. Next, participants were asked to assess the current price per unit. Then they were asked about their knowledge level $(1=$ little to $9=$ extremely high) concerning the prices of residential real estate in this area immediately after making their estimate. Moreover, they were asked to judge the knowledge level of other group members.

The third part served to collect demographic information about the participants.

\subsubsection{Results and Discussion}

In this experiment, we carried out a 2 (knowledge: high vs. low) X 2 (anchor: high vs. low) ANOVA analysis. As expected, significant interactive effects were generated between high and low knowledge on the one hand, and high and low anchors on the other $(F(1,156)=48.32, p<0.001)$. We simultaneously performed a simple F-test on Groups A and B respectively: in group B (that is, those without any house-buying experience and having no plans to buy a house in a year), we found a significant anchoring effect $(F(1,78)=98.97, p<0.001)$. Participants gave higher estimates following a high anchor $(M=55.15, S D=5.82)$ as compared to following a low anchor $(M=43.30, S D=4.79)$. In group A (that is, those who had house-buying experience within the past year), there was no anchoring phenomenon $(F(1,78)=0.14, p=0.708)$. The mean and standard deviation for those receiving high anchors and low anchors were $M=48.30, S D$ $=5.18$ and $M=47.88, S D=4.49$, respectively.

The results of Study 1 indicate that in a specific domain, although the participants were asked about their knowledge level immediately after making their estimate, those with relatively more experience will certainly increase their level of knowledge in this domain. Therefore, their knowledge judgments could have re- 
flected their confidence in their estimates more than their knowledge about the topic [14]. However, simultaneously, we also used the method of mutual rating of each other's level of knowledge to re-verify the results. We found that, regardless of the scores given by the participants concerning their own level of knowledge or those given by other groups concerning their own group, the mean score of participants with experience are both significantly higher than that of those without experience (self-assessment score $F(1,158)=266.17, p<$ $0.001)$; peer assessment score $\mathrm{F}(1,158)=49.97, p<0.001)$. In general, if the assessment of knowledge level is based, to a significant extent, on self-confidence, then the score given to others will be relatively lower. However, this did not happen when we asked participants to assess their own and others' knowledge levels, which means that, regardless of whether they were making an assessment of themselves or others, the score obtained by those with experience was significantly higher than that of those without experience.

Regarding these results, we believe that those with lower knowledge, in their quantitative estimation (housing prices), might rely on the plausible anchors given by us as their starting value for the estimation. Furthermore, due to an insufficient adjustment, those receiving high anchors would give a relatively higher estimation of the price and those receiving low anchors would give a relatively lower estimation. In the high knowledge group, when making their price estimation, they might disregard the anchors given by us and use their own knowledge accumulated from recent experience as the reference value for the price estimation. That is to say, if they have conducted sufficient price inquiries and comparisons over the past year related to purchasing a property, the anchors given would have little effect on them.

Our results prove the deduction of our first hypothesis: anchoring effects will not take place among people with higher knowledge, whereas they are inevitable among people with lower knowledge. The results of this study would become the foundation for our following studies, which would leave out those with lower knowledge and focus on the issue of conditions under which anchoring effects would still take place among those with higher knowledge.

\subsection{Study 2}

\subsubsection{Participants}

We successfully interviewed 160 professional residential real estate investors who had worked in this market for over 10 years, and specifically had more than 3 cases bargained per year in the last 5 years in Taipei City (Taiwan). We classified them as Group A (those with high knowledge). At the same time, we also successfully interviewed 160 ordinary residents of Taipei City. They had never had the experience of buying a house, and had no plans to do so within the coming year. We classified them as Group B (those with low knowledge). All participants would receive a cup of black tea as a reward, and we would further select five participants by lot, with each receiving a 7-eleven NT\$100 gift certificate. 


\subsubsection{Materials}

Similar to Study 1, our interviews were also divided into three parts. In the first part, we first invited the participants of group A to share their investment experience in real estate. For Group B participants, we asked them to share their views on the current real estate market. The second part was an anchoring test. The third part was conducted for collecting demographic information about the participants.

\subsubsection{Procedure}

As the contents of the first and third parts of these interviews were similar to Study 1, we did not repeat the explanation here. They were in line with our conclusions." When faced with a complicated and difficult problem that cannot be resolved within a short time frame ..." Therefore, this experiment was divided into two stages. In the first stage, we adapted the same approach as in study 1 and ask all our respondents to value the properties we offered. Since this stage was consistent with the practice of study 1 , we no longer provided detailed descriptions. The only difference lay in the fact that after the valuation, we invited our subjects to rate the complexity and difficulty of the problem $(1=$ little to $9=$ extremely high). This score was mainly used to compare the complexity and difficulty of the topics we provided in the second stage. The second stage was divided into two parts. In the first part, we gave the subjects 30 minutes to answer the question (no time pressure), and with only 1 minute in the second part (time pressure). We randomly assigned subjects from groups A and B to the first and second part. Except for the time limits, the questions in both parts were exactly the same. In this stage, we designed a more complicated question. We presented the participants with two sets of photos about a residential real estate property. We told the participants that the first set of photos showed the house bought by an investor during the last 2 years. Besides showing by photos of the then conditions of the house, we offered information about the house, including its location, size, type of building, and years. After that, we presented a second set of photos. The house in this set of photos was actually the same as the one in the first set, but its exterior and interior had been renovated and it was newly furnished as well. We told the participants that the investor had already sold this house in the previous month without telling them the purchase price 2 years earlier or the selling price in the last month, but we indicated to them that there were some hidden expenses both in owning the house and in the transaction process, such as transaction taxes, agency fees, interior decoration fees, bank loan interest, and so on. We simply indicated the related expenses, but we did not clearly tell the participants the actual amount of these expenses. Finally, we invited the participants to assess whether the profits obtained by the investor in this transaction were higher or lower than New Taiwan dollar 5 million (high anchor versus 500 thousand: low anchor). Next, the participants were asked to assess how much the investor had gained in this bargain. The main reason we made this hint regarding the cost of real estate transactions was our hope that the two groups would have a uniform basis for estimation, avoiding the situation 
where Group B participants only based the estimated value we wanted on the price difference between buying and selling, ignoring the costs and fees associated with such transactions, which would create disparity in the basis of estimation with Group A participants, thus causing the estimated value we acquired to lose their referentiality. As with Study 1, we then asked all participants to rate the level of their knowledge of this transaction we provided ( $1=$ little to $9=\mathrm{ex}$ tremely high). Moreover, they were asked to judge the knowledge level of other group members. Finally, we asked our subjects to rate the complexity and difficulty of the questions and the stress associated with given time constraint $(1=$ little to $9=$ extremely high). While inquiring about complexity, difficulty and time pressure, we used [24]: "Overall, how difficult was this task?" and "How much time pressure did you feel while working on this task?' When rating and in order to maintain the consistency of the overall experiment, we used a grading scale of 1 to 9 .

\subsubsection{Results and Discussion}

Before we proceed to the result analysis, let us explain the premises of our hypothesis. First, we compared the scores of all the subjects' grading complexity and difficulty in the first and second stage. The results show that when asked questions about the investor's profit on a given transaction (stage 2) and property valuation (stage 1), the complexity and difficulty of the questions experienced by stage 2 subjects were significantly higher than those experienced by stage 1 subjects $(F(1,638)=4.333, p<0.001)$. In other words, this experiment effectively manipulates both complexity and difficulty. When evaluating time pressure, the pressure experienced by the subjects who only had 1 minute to respond (time pressure) was significantly higher than that of the subjects who had 30 minutes (no time pressure) to do so $(F(1,318)=2.581, p<0.001)$. In the absence of time limits, the average response time amounts to 13 minutes and 2 seconds. Therefore, subjects granted 30 minutes could answer calmly and without any time pressure. This result shows that we are also successful at time pressure manipulation. We must also note that even though we asked subjects to evaluate given properties in the first stage, neither the anchor nor the knowledge levels will be analyzed any further because the same experiment has been already concluded in study 1.

Let us proceed to the final analysis. In stage 2 of this experiment, we found that in the absence of time pressure, there are significant interactions between knowledge and anchors $(F(1,156)=154.53, p<0.001)$; In the presence of time pressure there is no significant interactions $(F(1,156)=1.709, p=0.193)$. Although, when facing difficulties, knowledgeable participants rely on their knowledge as the basis for the estimation, they are not affected by any anchors. However, when facing a complex scenario and making an estimation in a short time, the level of knowledge (or having or not having) was always influenced by the given anchor.

This result agrees with our conjecture: when given a short period of time to make a relatively more complicated and difficult quantitative estimation, those 
with higher knowledge, for fear of not able to complete their estimation within a fixed period of time, would take the anchors we gave them despite their past experience might have told them what the actual profits should be in this kind of situation. However, to arrive at an answer in a short period of time, they might have been more inclined to make their estimation based on the anchors we provided. Hence, the anchoring effect occurred. We must also note that regarding the assessment of the knowledge of the content of the questions of this study, similar to Study 1, regardless of whether the assessment was by self or the other group, the scores of people with high knowledge were significantly higher than those with low knowledge (self-assessment: $F(1,318)=2.106, p<0.001$; peer assessment: $F(1,318)=2.120, p<0.001$.).

\subsection{Study 3}

\subsubsection{Participants}

We successfully interviewed 120 financial specialists with more than 10 years' experience at banks, whose main duties were managing customers' assets and the sales of financial products, such as insurance, funds, bonds, and so on. To be qualified for this job, they were required to register in the related fields. Also, during their employment, they were required to receive training for more than 10 hours every week, and possess a certain level of knowledge of the pulse of international finance. We classified these people as Group A (high knowledge). We also interviewed 120 ordinary people, who did not work in the fields of finance and securities, and who did not regularly invest in stocks or funds; we classified these people as Group B (low knowledge). All participants would receive a small notebook as reward.

\subsubsection{Materials}

The contents of the interviews comprised three parts. For Group A, we mainly asked them to share their past experience, both in their work and in helping clients in managing their investment and finance in the first part. For Group B, the first part mainly involved inviting participants to express their views on the current financial situation. The second was an anchor test. The third part was conducted for collecting demographic information about the participants.

\subsubsection{Procedure}

The contents and research of the first part had the same intention as that of Studies 1 and 2 and were not directly related to this study, hence requiring no further explanation. The second part was an anchoring test, which is also the main component of this study. Regarding the anchor test, it was conducted in two stages. In the first stage, there were 60 financial experts (members of Group A) and 60 ordinary people (members of Group B) participating in our experiment. Before administering the anchoring question, we first administered a test with nine questions (see Table 1), and told them that if they could correctly answer five or more of them, they would receive an extra prize. Then we proceeded with the anchor test. We adopted a counterbalance measure to prevent our results 
Table 1. The list of nine questions.

1. Who currently serves as the Governor of the Central Bank of the Taiwan?

2. Who is the current chair of the Board of Governors of the US Federal Reserve System?

3. What is the term of office for the Governor of the Central Bank of Taiwan?

What is the term of office of the chair of the Board of Governors of the US Federal Reserve System?

5. To boost its exports, what usually is the exchange rate policy of a country?

6. What does NAFTA stand for?

7. What is the main currency currently in circulation in France?

8. According to what the International Monetary Fund announced in 2015, which countries were the three largest economies in the world?

9. Is exchange rate depreciation beneficial or harmful to exports?

from being affected. First, we informed the participants that some people (without saying who they were) believed that the probability that in 2016, Taiwan's GDP growth rate reaching $1.2 \%$ would be $65 \%$ (high anchor point versus $25 \%$ : low anchor point). We asked the respondents if they believed that the chance of Taiwan's GDP growth rate reaching $1.2 \%$ in 2016 was higher or lower than $65 \%$ (high anchor versus 25\%: low anchor), and then asked them to assess this probability. After they gave their answers, we invited them to assess the reliability of the source of this information on a scale that ranged from 1 (=extremely unreliable) to 9 (=absolutely reliable). Then, they were asked about their knowledge level ( $1=$ little to $9=$ extremely high) concerning this issue immediately after they made their estimate.

In the second stage, 60 financial experts (members of Group A) and 60 ordinary people (members of Group B) participated in our experiment. Like in the first stage, we first gave them a test with nine questions, and also told them that those who correctly answered five or more questions would receive an extra prize. We then administered the anchor test. We verbally narrated a report, whose gist was an analysis of Taiwan's current economic situation and prospects. Simultaneously, we reminded the participants of the source of this report, telling them that in this report, an authoritative expert (an economic commentator) believed that the probability of Taiwan's GDP growth rate reaching $1.2 \%$ in 2016 would be $65 \%$ (high anchor point versus $25 \%$ : low anchor point). We again asked the respondents if they believe that the chance of Taiwan's GDP growth rate reaching $1.2 \%$ in 2016 is higher or lower than $65 \%$ (high anchor versus 25\%: low anchor), and then asked them to assess this probability. Just as in the first stage, after finishing the anchoring questions, we also invited the participants to assess the reliability of the source of this information as well as their knowledge level of this issue using the same assessment method as in stage 1.

\subsubsection{Results and Discussion}

Just like in study 2, prior to performing our anchoring analysis, we first explained to the participants the confidence levels of our sources of knowledge and 
anchors. First, regarding the reliability of information sources of the anchors, the participants of the second stage had significantly greater confidence in the information source we provided than those of the first stage, $F(1,238)=573.54, p<$ $0.001(M=5.91$ vs. 2.66). That is to say, our manipulation of the confidence level of the information source of the anchors was successful. Next, regarding the knowledge test, the average number of correct answers of Group A to our questions on international finance (those with high knowledge) was significantly higher than that of Group B (those with low knowledge), $F(1,238)=727.07, p<$ 0.001 ( $M=5.99$ vs. 2.58 ). Secondly, in the self-assessment of all participants regarding their knowledge levels, the average score of Group A members was also significantly higher than that of Group B members, $F(1,238)=853.81, p<0.001$ ( $M=7.08$ vs. 3.37). Lastly, we conducted an analysis of the correlation between the number of the participants' correct answers and the scores of their self-assessment, their correlation coefficients in these two items were $r=0.68$ and $r=0.50$ respectively.

Next came our anchoring analysis. In the first stage, we carried out a 2 (knowledge: high vs. low) X 2 (anchoring: high vs. low) two-way ANOVA analysis. The results showed that when the participants had lower confidence in the information sources of the anchors (which is what we noted in Study 1, "in general situations"), there was significant interaction between knowledge and anchors, $F(1,116)=34.78, p<0.01$. But in the second stage, when the reliability of the sources of the anchors the participants received was higher, there was no significant interaction between knowledge and anchors $(F(1,116)=0.36, p=$ $0.55)$.

In line with what we conjectured, even for the participants with higher knowledge, when faced with an opinion they considered to be authoritative, they would still choose to believe it and make an estimation close to this opinion. When in the first stage the participants were told that it was "some people's opinion" on the probability of Taiwan's GDP growth rate reaching 1.2\% in 2016, they tended to rely more on their own knowledge accumulated through experience; hence, they would not take the given anchors as the reference value in making their quantitative estimation, but rather make their final decision according to judgments based on their own knowledge. However, when it came to an opinion of a person of great authority, they might then consider it (as they might believe it to be more reliable than their own knowledge) and might be less concerned with their past understanding of this same issue. The results of this study have proven our third hypothesis: anchoring effects will still occur in people with greater knowledge when the source of information of the anchors possesses a high degree of reliability.

Although the experimental results conformed to our expectations, we must note that in this experiment, what we obtained was only when the information source of anchors was reliable, the anchoring effect would still occur in people with high knowledge. However, we have no way knowing how high the level of reliability should reach before it renders knowledge ineffective in anchoring. 


\subsection{Study 4}

\subsubsection{Participants}

We successfully interviewed 120 staff members, who worked as credit account managers in banks, and who became subjects of our experiment. Before advancing to a position of a credit account manager, they had to have at least 3 years of experience at entry level (as an advisor or credit account assistant), and had their skills verified by a respective bank supervisor. Afterwards, they became eligible for a three-month training course, and finally obtained a certificate from the Taiwan Academy of Banking and Finance Institute, which allowed them to officially hold this position. In this experiment, participants from group A had over 10 years of experience as credit account managers (more practical experience), and group B participants had between 1 and 3 years of experience (less practical experience). Participants would all receive a cup of black tea as a reward.

\subsubsection{Materials}

Our interviews were divided into three parts. In the first part, participants were asked to share their work experience. This part was not related to the information we wanted to analyze, but was mainly conducted to arouse the interest of the participants on the issues, with the hope that they would focus more on the following anchor questions. The second part was an anchor test. The third part was conducted for collecting demographic information about the participants.

\subsubsection{Procedure}

As the first and third parts of the questionnaire were the same as those in Study 1 , we did not repeat them. Part 2 is about the anchor test. We provided our subjects with 2 questions. In the first question, we provided the basic information about a fictional company: its activities, size, operational profile, current business loans and account balance. Then we asked our subjects the following question: if the company was to apply for a short-term loan in the amount of NTD 30 million, would the probability of having it approved be higher or lower than $65 \%$, according to your experience and judgement (high anchor versus $25 \%$ : low anchor)? Then we ask our subjects to assess how likely the case is to be approved. And finally, we ask them to assess their practical experience in dealing with new clients in their own career $(1=$ little to 9 extremely high). In the second question, we followed the steps of the previous one, and gave relevant information about another company. Then, we informed our subjects that the company's loan was due but it could not be paid because of insufficient funds. The company was short of funds, which resulted in a late loan payment ... which eventually was paid on time. Subsequently, we asked our subjects if the probability of the company eventually calling a credit default is higher or lower than $65 \%$, according to their experience (high anchor versus 25\%: low anchor. Then, we asked them how likely the default was to happen. Just like in the previous instance, we asked them to assess their own practical experience ( $1=$ little to 9 extremely high). At the end of the questionnaire, we asked our subjects to assess their knowledge as credit account managers $(1=$ little to 9 extremely 
high). Counterbalancing the anchors did not affect the results. In other words, for some of the subjects with the two questions the high anchor occurred twice, for some the low anchor occurred twice, and of course, there were also people who got both a low and high anchor. We use 2 questions to test our subjects separately, and see whether experience and anchors influence each other.

\subsubsection{Results and Discussion}

The main reasons why the test consists of two questions are as follows: Firstly, we believe that the anchoring effect comes into play when those who have professional knowledge encounter a problem which cannot be solved with the mere help of that very knowledge, or when they lack sufficient practical experience to make the calculation. This situation should occur in all aspects of the entire process, not only in part of it. Therefore, we have raised two questions from their field of work. One concerns finding new customers, while the other maintaining customer relationships. From the practical point of view, the judgement in these two cases should rely on practical experience Knowledge alone is not enough. We hope that our conclusion applies to the entire process, and as long as there is a lack of practical experience, not even professional knowledge can prevent the anchoring effect. Moreover, the result of this twofold test will strengthen our conclusion, and get verified in different professional fields. Secondly, when we compare our estimations with regular estimations, we might get more answers influenced by subjects' personal characteristics. For example, when our subject's personality is optimistic, the success rate of new applications may be estimated to be high, and that of credit defaults to be low. If the subject is conservative by nature, the results may be opposite. Therefore, in this twofold test we randomly assign high and low anchors to reduce the impact of this factor on the results of our experiment. Generally speaking, our expectation was that knowledgeable participants with abundant experiences in this domain should not be influenced by anchoring. For those with only knowledge but lack relatively abundant experiences, anchoring effects would still come into play in their numeric estimate. As expected, regardless of how likely the new credit application is to succeed or how likely the credit default is to be called, the mutual influence of different experience and anchor levels in our subjects is significant $(F(1,116)=14.42, p<0.001) ; F(1,116)=14.28, p<0.001)$, respectively). In other words, the results of the two different parts of the test are as we expected. Having taken into account the personal traits of our subjects and assigned random anchors, we noticed they did not affect the results of our experiment. As for the individual self-assessment of experience, we found that in the twofold test group A, subjects scored significantly higher than group B $(F(1,118)=55.72, p<$ $0.001) ; F(1,118)=62.43, p<0.001)$, respectively). In the self-assessment of knowledge, the scores of the two groups had no significant differences $(F(1,118)$ $=1.242, p=0.267$ ).

The situation of "armchair strategy" is not uncommon in daily life. In other words, there are always discrepancies between reality and the decisions made by people possessing only theoretical knowledge but lacking practical experience. In 
this test, we have demonstrated that in general situations, those with higher knowledge may not be influenced by anchoring, but if they lack relatively abundant experiences, the anchoring effect will occur.

\section{General Discussion}

We designed four tests to demonstrate the relation between knowledge and anchoring. Many studies have already put forward their findings on this issue, one of which is an investigation of the anchoring effects in the domain of American football by [11], suggesting that participants with higher knowledge exhibit smaller anchoring effects. However, the focus of the present study is on identifying under what kinds of conditions anchoring effects will exert on those people with higher knowledge.

Study 1 served as the foundation for our several follow-up studies. We found that when people have a relatively high level of knowledge, anchoring effects will definitely not come into play, but for those lacking knowledge, such effects will undoubtedly occur. In Study 2, we used a two-stage experiment to estimate the complexity and difficulty of the problem. The first stage is the same as study 1 , but in the second stage, we increased the complexity and difficulty of the problem. Moreover, in the second stage, we divided the subjects into two groups. The first was given sufficient answer time (no time pressure), while the other had one minute to answer (time pressure). The results show that when faced with more complex and difficult tasks without any time pressure, the high/low knowledge-anchor interactions are insignificant. In other words, even if knowledgeable people are faced with difficult problems, but have sufficient time, they rely on their knowledge to make calculations, and no anchor effect occurs. However, when time pressure is present, in order to answer in time, the knowledgeable subjects rely on the provided anchors to make initial calculations. When time is insufficient, the anchoring effect comes into play. In Study 3, we indicated the source of information of the anchors. We found that, when the participants had relatively higher confidence in the source, the anchoring effect would occur. In Study 4, we distinguished the participants with higher knowledge in terms of the experience they had. We found that different amounts of practical experiences and high/low anchors influence each other. In other words, even within their field of expertise, when they have relatively little practical experience, the subjects' estimations are still affected by given anchors.

\subsection{Research Contributions}

Past researches on anchoring conducted by researchers in the past focused on verifying the existence of the anchoring effect [5] [6], whereas recently, researchers have begun to consider knowledge as a very important variable in the research regarding the anchoring effect [16] [17]. However, there has yet been any conclusion as to whether knowledge has any influence on anchoring. In this study, four experiments were conducted. Firstly, the conclusions drawn by scholars from the knowledge and anchoring research were verified once again 
using an estimation on real estate prices. The research result showed that generally knowledge tends to have an effect on anchoring. Secondly, we proposed a new hypothesis which had never been proposed by any scholars in the pastalthough knowledge does have an effect on anchoring, under certain circumstances, knowledge has no effect on anchoring. The anchoring effect also happens among highly educated people when they engage in number estimation.

The experiment results showed that the anchoring effect occurs among highly educated people when they engage in complex number estimation tasks which have a time limit, when the source of anchor information was highly credible, or when they lacked experience in that particular field. These study results can help clarify the role that knowledge plays in affecting the anchoring effect under certain circumstances, and provide future researchers with new research directions to study the anchoring effect.

\subsection{Limitations and Future Researches}

The main purpose of this study was to prove whether the anchors take effect among highly educated people, thus causing the anchoring effect. Although the hypothesis proposed had been proven by experiments, there still are research limitations in the experiments. The first limitation is how researchers measure knowledge. In the past, scholars tended to categorize the subjects into two groups, highly educated and not highly educated when examining the relationship between knowledge and anchoring. One of the methods they used was to classify people who work in a professional field as highly educated and those who do not as not highly educated. For example, [4] classified real estate agents as highly educated people and postgraduates as not highly educated people. The second limitation is that some scholars attempted to classify subjects by asking them to rate themselves on their level of knowledge such as in [11]. Some scholars believed the self-assessment approach might give more weight to the factor of self-confidence than the level of knowledge or experience of the subjects [14]. In this study, we took into account the subjects' professions as well as adopted the self-assessment approach to categorize subjects into two different groups, more highly educated and less educated. We also adopted the mechanism of mutual evaluation. However, we were unable to measure the actual level of knowledge of our subjects. Moreover, in our attempt to find out how knowledge affects the anchoring effect (Experiment 1), we could only define more highly educated people are less likely to be affected by anchors under general circumstances compared to those who are less educated. But we were not able to draw a conclusion as to how smart a subject has to be in order for the relationship between knowledge and anchoring to become apparent.

The second issue is that the answers implied in the number estimation using real estate prices as anchors were uncertain. Real estimate prices were fluctuant, so there was no fixed answer. Therefore, in the experiment, the subjects might have been influenced by the recent reports or evaluation on real estate, thus having a preconception of the prices prior to the experiment instead of evaluat- 
ing the scenario based on a random anchor provided. In this experiment, we did not propose any possible variable for discussion, which was another limitation in this study.

Thirdly, the richness of experience might be a subjective judgment. Generally, the more experience one has in a specific field, the knowledge the person has. In this study, we considered the number of years of experience each experimental subject has in the field, in conjunction with the self-assessment completed by the subject and their peer review to determine the level of their knowledge. The variable of "experience" was not quantitatively tested. For this reason, experience and knowledge might overlap.

The recent studies on anchoring mostly centered on the role of knowledge, which leaves a lot of unanswered questions as to whether knowledge has an effect on anchoring. This study first proposed that knowledge generally has an effect on anchoring except in special scenarios when the factor of knowledge does not take effect. Nevertheless, this study found out three scenarios where knowledge does not have an effect on anchoring. In future studies, more relevant scenarios might be found to prove the significant role of knowledge in influencing the anchoring effect and to clarify the relationship between knowledge and anchoring.

\section{Conclusion}

Past studies have indicated that anchoring seems to be a stable psychological phenomenon. However, many scholars have attempted to find a factor that can moderate anchoring effects, and a person's level of knowledge has become the focus in recent studies by scholars. Why might the level of knowledge moderate the anchoring phenomenon? This is because people with greater knowledge should have a better understanding of the actual conditions or answers [11] related to specific matters than the average person. We have also adopted a positive view of the relation between anchoring and knowledge, meaning that knowledge can indeed moderate anchoring effects. However, we also believe that such moderation will only occur in general situations. When high-knowledge people are faced with a complex quantitative estimation that must be made within a short period of time, they will still be unable to escape the influence of anchoring. In addition, we believe that, if the information source of the anchors is given and when high-knowledge participants have a relatively high degree of confidence in the source, then anchoring effects will occur. Finally, we have also shown that, even if one possesses relatively greater knowledge, but in those domains where one's experience is relatively meager, anchoring effects will still occur.

\section{References}

[1] Jacowitz, K.E. and Kahneman, D. (1995) Measures of Anchoring in Estimation Tasks. Personality and Social Psychology Bulletin, 21, 1161-1166.

https://doi.org/10.1177/01461672952111004 
[2] Mussweiler, T. and Strack, F. (2000) Numeric Judgment under Uncertainty: The Role of Knowledge in Anchoring. Journal of Experimental Social Psychology, 36, 495-518.

[3] Strack, F. and Mussweiler, T. (1997) Explaining the Enigmatic Anchoring Effect: Mechanisms of Selective Accessibility. Journal of Personality and Social Psychology, 73, 437-446. https://doi.org/10.1037/0022-3514.73.3.437

[4] Northcraft, G.B. and Neale, M.A. (1987) Experts, Amateurs, and Real Estate: An Anchoring-and-Adjustment Perspective on Property Pricing Decisions. Organizational Behavior and Human Decision Processes, 39, 84-97.

[5] Cervone, D. and Peake, P.K. (1986) Anchoring, Efficacy, and Action: The Influence of Judgmental Heuristics on Self-Efficacy Judgments and Behaviors. Journal of Personality and Social Psychology, 50, 492-501. https://doi.org/10.1037/0022-3514.50.3.492

[6] Plous, S. (1989) Thinking the Unthinkable: The Effects of Anchoring on Likelihood Estimates of Nuclear War. Journal of Applied Social Psychology, 19, 67-91. https://doi.org/10.1111/j.1559-1816.1989.tb01221.x

[7] Englich, B., Mussweiler, T. and Strack, F. (2006) Playing Dice with Criminal Sentences: The Influence of Irrelevant Anchors on Experts' Judicial Decision Making. Personality and Social Psychology Bulletin, 32, 188-200. https://doi.org/10.1177/0146167205282152

[8] Chapman, G.B. and Johnson, E.J. (1994) The Limits of Anchoring. Journal of Behavioral Decision Making, 7, 223-242. https://doi.org/10.1002/bdm.3960070402

[9] Smith, A.R. and Windschitl, P.D. (2011) Biased Calculations: Numeric Anchors Influence Answers to Math Equations. Judgment and Decision Making, 6, 139-146.

[10] Galinsky, A.D. and Mussweiler, T. (2001) First Offers as Anchors: The Role of Perspective-Taking and Negotiator Focus. Journal of Personality and Social Psychology, 81, 657-669. https://doi.org/10.1037/0022-3514.81.4.657

[11] Smith, A.R., Windschitl, P.D. and Bruchmann, K. (2013) Knowledge Matters: Anchoring Effects Are Moderated by Knowledge Level. European Journal of Social Psychology, 43, 97-108. https://doi.org/10.1002/ejsp.1921

[12] Epley, N. and Gilovich, T. (2004) Are Adjustments Insufficient? Personality and Social Psychology Bulletin, 30, 447-460. https://doi.org/10.1177/0146167203261889

[13] Wilson, T.D., Houston, C.E., Brekke, N. and Etling, K.M. (1996) A New Look at Anchoring Effects: Basic Anchoring and Its Antecedents. Journal of Experimental Psychology, 125, 387-402. https://doi.org/10.1037/0096-3445.125.4.387

[14] Englich, B. (2008) When Knowledge Matters-Differential Effects of Available Knowledge in Standard and Basic Anchoring. European Journal of Social Psychology, 38, 896-904. https://doi.org/10.1002/ejsp.479

[15] Englich, B. and Soder, K. (2009) Moody Experts-How Mood and Expertise Influence Judgmental Anchoring. Judgment and Decision Making, 4, 41-50.

[16] Smith, A.R. and Windschitl, P.D. (2015) Resisting Anchoring Effects: The Roles of Metric and Mapping Knowledge. Memory \& Cognition, 43, 1071-1084. https://doi.org/10.3758/s13421-015-0524-4

[17] Cheek, N.N., Coe-Odess, S. and Schwartz, B. (2015) What Have I Just Done? Anchoring, Self-Knowledge, and Judgments of Recent Behavior. Judgment and Decision Making, 10, 76-85.

[18] Klayman, J. and Ha, Y. (1987) Confirmation, Disconfirmation, and Information in Hypothesis Testing. Psychological Review, 94, 211-228.

https://doi.org/10.1037/0033-295X.94.2.211 
[19] Tversky, A. and Kahneman, D. (1974) Judgment under Uncertainty: Heuristics and Biases. Science, 185, 1124-1131. https://doi.org/10.1126/science.185.4157.1124

[20] Mussweiler, T. and Strack, F. (1999) Hypothesis-Consistent Testing and Semantic Priming in the Anchoring Paradigm: A Selective Accessibility Model. Journal of Experimental Social Psychology, 35, 136-164.

[21] Wong, K.F.E. and Kwong, J.Y.Y. (2000) Is $7300 \mathrm{~m}$ Equal to $7.3 \mathrm{~km}$ ? Same Semantics but Different Anchoring Effects. Organizational Behavior and Human Decision Processes, 82, 314-333.

[22] Mussweiler, T. and Englich, B. (2003) Adapting to the Euro: Evidence from Bias Reduction. Journal of Economic Psychology, 24, 285-292.

[23] Ross, L., Lepper, M.R. and Hubbard, M. (1975) Perseverance in Self-Perception and Social Perception: Biased Attributional Processes in the Debriefing Paradigm. Journal of Personality and Social Psychology, 32, 880-892. https://doi.org/10.1037/0022-3514.32.5.880

[24] Crescenzi, A., Cupra, R. and Arguello, J. (2013) Time Pressure, User Satisfaction and Task Difficulty. Proceedings of the American Society for Information Science and Technology, 50, 1-4. https://doi.org/10.1002/meet.14505001121

\section{Scientific Research Publishing}

Submit or recommend next manuscript to SCIRP and we will provide best service for you:

Accepting pre-submission inquiries through Email, Facebook, LinkedIn, Twitter, etc. A wide selection of journals (inclusive of 9 subjects, more than 200 journals) Providing 24-hour high-quality service User-friendly online submission system Fair and swift peer-review system Efficient typesetting and proofreading procedure Display of the result of downloads and visits, as well as the number of cited articles Maximum dissemination of your research work

Submit your manuscript at: http://papersubmission.scirp.org/ Or contact jss@scirp.org 